\title{
Closed-loop Adaptive Filtering for Supressing Chest Compression Oscillations in the Capnogram During Cardiopulmonary Resuscitation
}

\author{
Mikel Leturiondo ${ }^{1}$, J.J. Gutierrez ${ }^{1}$, Sofía Ruiz de Gauna ${ }^{1}$, Sandra Plaza ${ }^{1}$, José F Veintemillas ${ }^{2}$, \\ Mohamud Daya ${ }^{3}$ \\ ${ }^{1}$ University of the Basque Country (UPV/EHU), Bilbao, Spain \\ ${ }^{2}$ Emergentziak Osakidetza (Basque Health Service), Basque Country, Spain \\ ${ }^{3}$ Oregon Health \& Science University (OHSU), Portland OR, USA
}

\begin{abstract}
Capnography is widely used by the advanced-lifesupport during cardiopulmonary resuscitation (CPR). Continuous analysis of the capnogram allows guidance of adequate ventilation rate, currently $10 \mathrm{breaths} / \mathrm{min}$ for intubated patients. We used 60 out-of-hospital cardiac arrest episodes containing both clean and CC corrupted capnograms. Chest compressions (CC) induce highfrequency oscillations in the capnography waveform impeding reliable detection of ventilations. Thus, a clean capnogram is essential for a better ventilation detection performance. To clean the capnogram, an adaptive noise cancellation notch filter was designed using a Least Mean Square algorithm to minimize filtering error. A fixedcoefficient low-pass filter was optimized for comparison. For the whole test set, global Se/PPV improved from 93.0/92.2\% to 97.6/96.2\% after adaptive filtering and to 97.7/94.8\% after fixed-coefficient filtering. For the clean subset, Se/PPV maintained stable and for the corrupted subset, Se/PPV improved from 84.8/84.0\% to 95.2/92.7\% and $95.4 / 90.3 \%$, respectively. Filtering allowed reliable automated detection of ventilations in the capnogram even in the presence of $C C$ oscillations during $C P R$. Nevertheless, further evaluation of these techniques in large datasets is warranted.
\end{abstract}

\section{Introduction}

Cardiac arrest is one of the main causes of death in developed countries. Patient survival to cardiac arrest is related to several factors. The most important is the early start of cardiopulmonary resuscitation (CPR) which combines chest compressions and ventilations. Hyperventilation is common during CPR for both inhospital and out-of-hospital cardiac arrests even among highly trained rescuers. Although resuscitation guidelines recommend providing 10 breaths per minute to intubated patients, some clinical studies have documented ventilation rates over 30 breaths per minute [1], which have been shown to impair hemodynamics and worsen outcomes at cardiac arrest.

The latest resuscitation guidelines recommend the use of capnography waveform to monitor ventilation rate during CPR [2]. A clean capnogram is essential for a reliable analysis of patient response (Figure 1a). However, chest compressions (CC) administered to the patient frequently induce high-frequency oscillations (Figure 1b,c,d) in the capnogram [3] impeding reliable detection of ventilations [4].
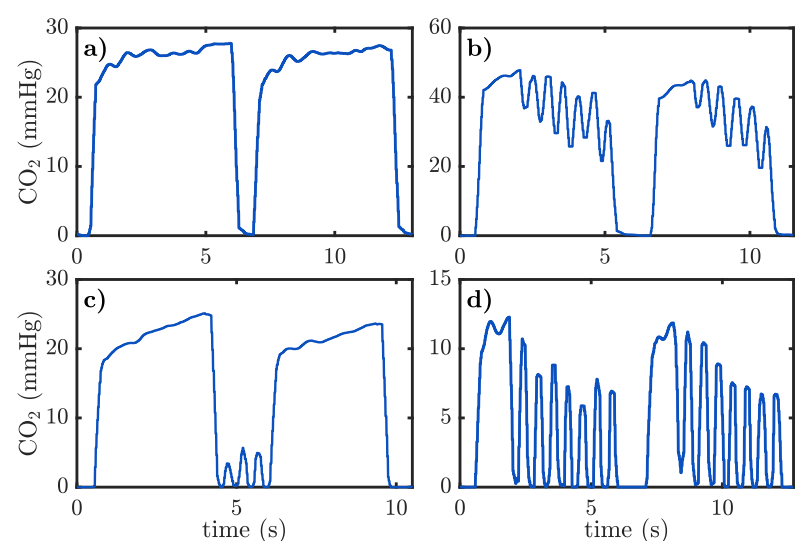

Figure 1. OHCA capnogram segments. a) Clean capnograms; b,c,d) Corrupted capnograms with CC oscillations in the plateau, baseline and form the plateau to the baseline, respectively.

In this context, the present paper describes the observational study performed to assess the performance of an adaptive closed-loop filter for suppressing CC oscillations in the capnogram during CPR. For this aim, we used a previously recorded out-of-hospital cardiac arrest (OHCA) registry. 


\section{Materials and methods}

\subsection{Database description and annotation}

The dataset analysed in this study was a subset (60 episodes) of a large OHCA registry collected and maintained by Tualatin Valley Fire \& Rescue (TVF\&R), an advanced life support first response Emergency Medical Services (EMS) agency in Oregon (USA). Episodes were recorded using Heartstart MRx monitor-defibrillators (Philips Medical Systems, Andover, MA, USA), equipped with a real-time CPR feedback system (QCPR, Laerdal Medical, Norway). Three concurrent signals were extracted: the transthoracic impedance (TI) signal, the compression depth (CD) signal and the capnogram.

For each episode, capnograms were time-shifted to compensate delay with respect $\mathrm{CD}$ and TI signals. A capnogram was classified as corrupted if evident CC oscillations appeared during more than $1 \mathrm{~min}$ of $\mathrm{CC}$ time.

Ventilation and CC instances were also annotated using the TI and the CD signal as the reference, respectively. TI signal was low-pass filtered ( $2^{\text {nd }}$-order Butterworth, cut-off frequency of $0.6 \mathrm{~Hz}$ ) to remove oscillations caused by $\mathrm{CC}$. Thus, slow oscillations caused by ventilations could be better observed in the filtered TI. Figure 2 depicts an example of the annotation process. Ventilations were annotated in the position associated to the inspiration onset (Figure 2, vertical lines) corresponding to a rise in the TI. $\mathrm{CC}$ instances were annotated in every relative maxima (Figure 2, red dots) corresponding to the maximum depth reached for each chest compression.
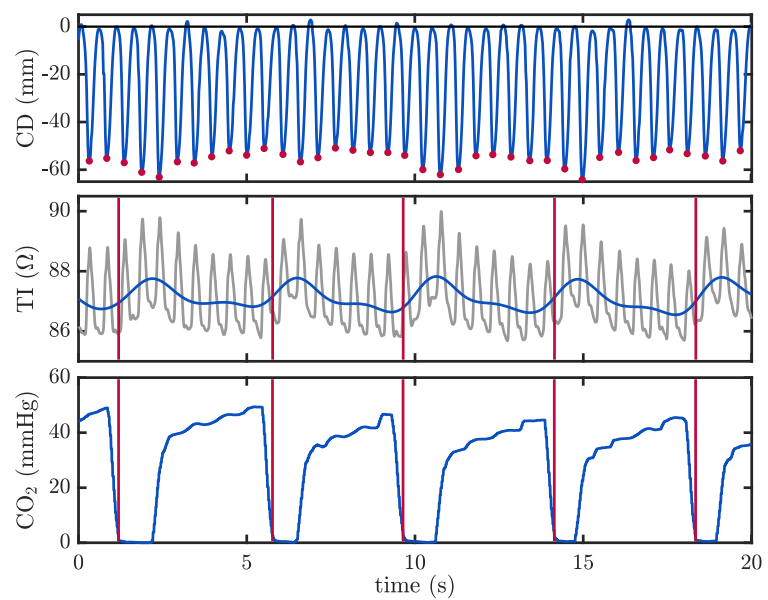

Figure 2. Example of ventilation and $\mathrm{CC}$ instance annotation.

Resulting ventilation annotations were our goldstandard to test the reliability of the ventilation detection algorithm. Meanwhile, CC instances were used to control the configuration of the adaptive closed-loop filter. Clean and corrupted capnograms were randomly and equally split into a training and a test set.

\subsection{Closed-loop adaptive filtering}

Quality of the recorded capnogram is essential for a reliable analysis. However, a clean capnogram, where the different phases of the respiratory cycle are identifiable cannot always be observed during CPR [4,5]. Sometimes ongoing resuscitation efforts induce fast sinusoidal oscillations at different rates and with varying amplitude superimposed on the capnogram. The most common way to suppress this kind of artefact consist of using a notch filter. Nevertheless, in this study an adaptive noise cancellation notch filter is proposed, adjusting the filter to the same frequency and phase of the artefact [6]. A Least Mean Square (LMS) algorithm has been used. The main objective of this algorithm is to minimize the error sequence power $(e[n])$ produced between the filter response $(y[n])$ and the desired response $(d[n])$.

The LMS filter, shown in Figure 3 , is based on estimations, so it achieves an error power $J(\infty)$ higher than the minimum error power $\left(J_{\min }\right)$ reached by the Wiener solution. In order to control the coefficient correction applied from a previous iteration to the next one, the LMS algorithm uses a parameter called step size $(\mu)$. When $\mu$ is high, the adjustment is fairly fast, but the difference between the final error power $J(\infty)$ and the minimum value $\left(J_{\min }\right)$ is higher than with lower $\mu$ values.

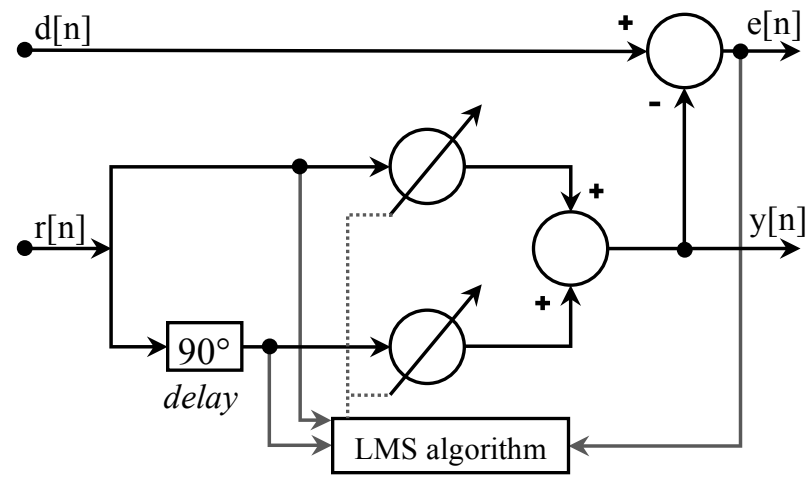

Figure 3. Adaptive noise cancellation LMS filter diagram.

Following the diagram depicted in Figure 3, the main input, $d[n]$, is the capnography signal with induced CC oscillations, while the reference input, $r[n]$, is a pure cosine $C \cdot \cos \left(\omega_{0} n+\theta\right)$, where $\omega_{0}=2 \pi f_{0} / f_{s}$ and $f_{0}$ is the instant frequency of the CC. When CC are provided, the cosine amplitude $C$ is ' 1 ', and ' 0 ' otherwise.

The output of the adaptive filter, $y[n]$ is an estimation of the artefact and the output of the noise cancellation system, $e[n]$, represents the capnography signal without induced $\mathrm{CC}$ oscillations. Using $e[n]$ and the reference signals the LMS algorithm adjusts the impulse response of the filter used in the next iteration.

The transference function (1) of this filter demonstrates that the noise cancellation system has the same properties 
of a notch filter designed to supress $\omega_{0}$. The unique design parameter of this adaptive filter is the $3 \mathrm{~dB}$ bandwidth (2).

$$
\begin{aligned}
& H(z)=\frac{z^{2}-2 z \cos \omega_{0}+1}{z^{2}-2\left(1-\mu C^{2}\right) z \cos \omega_{0}+1-2 \mu C^{2}} \\
& B W_{3 d B}=2 \mu C^{2}(r a d)=\frac{\mu C^{2}}{\pi T}(H z)
\end{aligned}
$$

\subsection{Performance evaluation}

We evaluated the performance of the adaptive filter in terms of its sensitivity ( $\mathrm{Se}$ ) and its positive predictive value (PPV) given by the ventilation detection algorithm and the gold-standard. Se was defined as the percentage of annotated ventilations that were correctly detected. PPV was defined as the percentage of detected ventilations that were correct. The maximum admissible tolerance for the position of the ventilation detection and the gold-standard annotation was $500 \mathrm{~ms}$.

We optimized the adaptive filter parameter $\left(B W_{3 d B}\right)$ with the training set to maximize Se while maintaining PPV above $92 \%$. A fixed-coefficient low-pass filter was designed and optimized ( $8^{\text {th }}$-order Butterworth, cut-off frequency of $1.5 \mathrm{~Hz}$ ) for comparison. The $95 \%$ confidence intervals $(95 \% \mathrm{CI})$ were computed for both metrics.

\section{Results}

Adaptive closed-loop filter optimization was achieved for $B W_{3 d B}=1 \mathrm{~Hz}$. For the training set (8102 ventilations), the Se and PPV were 98.4\% (95\%CI, 98.0-98.6) and 96.6\% (96.2-97.0), respectively.

Table 1 summarizes Se and PPV results for the LMS filter. Table 2 summarizes Se/PPV before filtering and after applying the fixed-coefficient filter. For the whole test set, global Se/PPV improved from 93.0/92.2\% to 97.6/96.2\% after adaptive filtering and to $97.7 / 94.8 \%$ after fixed-coefficient filtering. For the clean subset, Se/PPV maintained stable (99.8/99.1\% before, 99.8/99.1\% and 99.6/98.7\% after, respectively). For the corrupted subset, Se/PPV improved from $84.8 / 84.0 \%$ to $95.2 / 92.7 \%$ and $95.4 / 90.3 \%$, respectively.

Figure 4 shows the boxplots of $\mathrm{Se}$ and PPV values before and after filtering. For both fixed-coefficient and adaptive closed-loop the dispersion of Se and PPV was very low before as well as after filtering, in case of clean episodes. However, the dispersion of Se and PPV was quite relevant for corrupted episodes before filtering.
Table 1. Se and PPV for the test set after adaptive filtering. $\mathrm{n}$ : number of annotated ventilations.

\begin{tabular}{lccc}
\cline { 2 - 4 } & $\mathbf{n}$ & Se (95\% CI) & PPV (95\% CI) \\
\hline Whole set & 7195 & $97.6(97.3-98.0)$ & $96.2(95.7-96.6)$ \\
Clean & 3905 & $99.8(99.6-99.9)$ & $99.1(98.8-99.4)$ \\
Corrupted & 3290 & $95.2(94.4-95.8)$ & $92.7(91.8-93.6)$ \\
\hline
\end{tabular}

Table 2. Se and PPV for the test set before filtering (left) and after fixed-coefficient filtering (right).

\begin{tabular}{lcccc}
\cline { 2 - 5 } & \multicolumn{2}{c}{ Before } & \multicolumn{2}{c}{ Fixed-coefficient } \\
\cline { 2 - 5 } & Se(\%) & PPV(\%) & Se (\%) & PPV (\%) \\
\hline Whole set & 93.0 & 92.2 & 97.7 & 94.8 \\
Clean & 99.8 & 99.1 & 99.6 & 98.7 \\
Corrupted & 84.8 & 84.0 & 95.4 & 90.3 \\
\hline
\end{tabular}

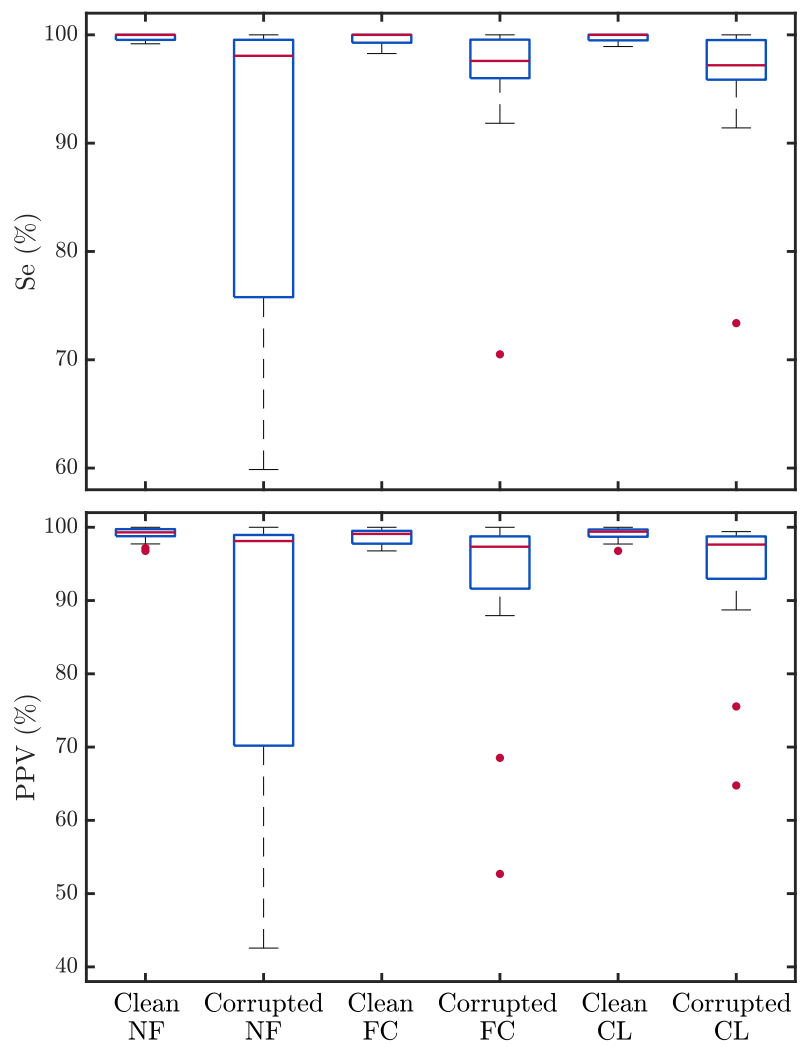

Figure 4. Se and PPV for the test set before filtering (NF, left), after fixed-coefficient filtering (FC, middle) and after closed-loop adaptive filtering (CL, right). Boxes show the median and IQR. Outliers are represented by dots 


\section{Discussion}

According to current resuscitation guidelines for advanced life support, ventilation rate monitoring during CPR is one of the recommended uses of the capnogram. However, the presence of high-frequency oscillations in the capnogram during $\mathrm{CC}$ may compromise a reliable detection of ventilations.

Our adaptive noise cancellation filter presented a very good ventilation detection performance. Thus, the results demonstrate that filtering the capnogram in case of clean episodes maintains good results of Se and PPV, and improve them in presence of artefact.

Comparing the results between adaptive and fixedcoefficient filtering, it seems that fixed-coefficient filtering would be enough. Nevertheless, due to CC rate variability, a study with a larger database is needed, while our adaptive filter is CD signal dependent, allowing us to adjust our filter to any kind of CC rate, but this requires using a CPR feedback system providing this signal.

Other studies proposing alternatives for ventilation detection with either the TI signal or the capnogram have also mentioned the signal limitations due to the presence of CC artefact [5]. Although the influence of CC artefact on the reliability of capnogram-based ventilation detection has been demonstrated [4], the suppression of this artefact has not been previously studied.

Our study has been the first to quantitatively characterize and measure the impact of an adaptive CC artefact suppression on OHCA capnograms. Our subsequent hypothesis is that automatic ventilation detection would improve if the capnogram waveform could be successfully restored. Designing new filtering approaches for this aim will be our next step, exploring different alternatives.

Our study has several limitations. First, the annotation of ventilations on the TI was not straightforward during CPR. We discarded some intervals because of unreliable TI signal (noise, disconnections) and filtering was needed to remove the $\mathrm{CC}$ artefact. No other reference signal was available as an alternative gold-standard. Finally, data came from a single monitor-defibrillator and so results may not be generalizable. We would need to characterize this further with other monitor-defibrillators.

\section{Conclusions}

Filtering allowed reliable automated detection of ventilations in the capnogram even in the presence of CC oscillations during CPR. Nevertheless, further evaluation of these techniques in large datasets is warranted given the variability of out-of-hospital CC and ventilation rates.

\section{Acknowledgements}

This work received financial support from the Basque Government (Basque Country, Spain) through the project IT1087-16 and the predoctoral research grant PRE-20161-0104. The authors thank the TVF\&R EMS providers for collecting and maintaining the data used in this study.

\section{References}

[1] Maertens VL, De Smedt LE, Lemoyne S, et al. Patients with cardiac arrest are ventilated two times faster than guidelines recommend: an observational prehospital study using tracheal pressure measurement. Resuscitation 2013; 84(7):921-926.

[2] Soar J, Nolan JP, Böttiger BW, et al. European Resuscitation Council guidelines for resuscitation 2015. Section 3. Adult advanced life support. Resuscitation 2015;95:100-147.

[3] Idris AH, Daya M, Owens P, et al. High incidence of chest compression oscillations associated with capnography during out-of-hospital cardiopulmonary resuscitation. Circulation 2010;122:A83.

[4] Leturiondo M, Ruiz J, Ruiz de Gauna S, González-Otero DM, Bastida JM, Daya M. A simple algorithm for ventilation detection in the capnography signal during cardiopulmonary resuscitation. Computing in Cardiology 2017. In press.

[5] Edelson DP, Eilevstjønn J, Weidman EK, Retzer E, Hoek TLV, Abella BS. Capnography and chest-wall impedance algorithms for ventilation detection during cardiopulmonary resuscitation. Resuscitation 2010;243 81(3):317-322.

[6] Widrow B, Stearns D. Adaptive signal processing. Prentice Hall. 1985

Address for correspondence.

Mikel Leturiondo Sota

mikel.leturiondo@ehu.eus

School of Engineering

Alameda Urquijo s/n, 48013-Bilbao (Spain) 